\title{
Prevalence of arrhythmias in dogs examined between 2008 and 2014
}

\author{
Agnieszka Noszczyk-Nowak, Marcin Michałek, Ewelina Kałuża, \\ Alicja Cepiel, Urszula Pasławska \\ Department of Internal Medicine and Clinic of Diseases of Horses, Dogs and Cats, Faculty of Veterinary Medicine, \\ Wroclaw University of Environmental and Life Sciences, 50-366 Wroclaw, Poland \\ marcin.michalek@upwr.edu.pl
}

Received: November 17, 2016 Accepted: March 8, 2017

\begin{abstract}
Introduction: The prevalence of arrhythmias in dogs and the influence of sex, breed, age, and body weight were analysed over a seven-year span. Material and Methods: In total, 1189 referrals for cardiological examination by electrocardiography were received at one academic centre in Poland between 2008 and 2014. The largest proportion of the examined dogs were crossbreeds with body weight below $25 \mathrm{~kg}(\mathrm{n}=153,12.87 \%)$, followed by German Shepherds $(\mathrm{n}=122,10.26 \%)$, Labrador Retrievers $(\mathrm{n}=68,5.72 \%)$, Yorkshire Terriers $(\mathrm{n}=63,5.3 \%)$, and Boxers $(\mathrm{n}=60,5.05 \%)$. Retrospective analysis was made of 1201 standing or right recumbent electrocardiograms without pharmacological sedation. The prevalence of arrhythmias was examined in terms of sex, age, body weight, and breed of the dogs. Results: A total of 630 (52.46\%) electrocardiograms showed no signs of arrhythmia, but $96(7.99 \%)$ and $475(39.55 \%)$ pointed to physiological and pathological arrhythmias respectively. The most commonly diagnosed type was atrial fibrillation with $33.68 \%$ incidence, followed by ventricular arrhythmias (28\%), sinus pauses (27.58\%), supraventricular arrhythmias (24\%), and atrioventricular blocks (22.95\%). Pathological arrhythmias were most commonly found in male dogs and in German Shepherds. Conclusions: Atrial fibrillation predominated, followed by premature ventricular complexes. Male dogs were generally more prone to heart rhythm disturbances.
\end{abstract}

Keywords: dogs, arrhythmia, atrial fibrillation, supraventricular arrhythmias, ventricular arrhythmias.

\section{Introduction}

Although the history of electrocardiography dates back to the $19^{\text {th }}$ century, the standard leads proposed at that time by Willem Einthoven are still in use nowadays. The superficial electrocardiogram still remains a relatively simple, widely available, accurate, and inexpensive method for evaluation of arrhythmias. Although it has been known for years, veterinary medicine still lacks a comprehensive and up-to-date analysis of the prevalence of heart rhythm disturbances in animals. Statistical analysis of arrhythmias in the general population of dogs have been published by several authors $(6,8,11,16)$, and studies describing cardiac dysrhythmias in specific breeds are also available (17). They suffer, however, from substantial limitations like outdated data and focus on a certain cardiac dysrhythmia or evaluation of a certain breed, making them difficult to compare. To the authors' best knowledge, the presented study was conducted on the largest animal group with heart rhythm disorders compared to previous investigations.

The aim of this study was to analyse the prevalence of heart rhythm disturbances in dogs and the influence of sex, breed, age, and body weight.

\section{Material and Methods}

Retrospective analysis included electrocardiographic recordings from 1189 dogs referred for cardiological examination with electrocardiography (ECG) at the Cardiology Unit between 2008 and 2014. Electrocardiographic recordings were obtained in a standing or right recumbent position, without pharmacological sedation, using a BTL-08 3-channel electrocardiograph (BTL Industries, U.K.). The causes for the animals to be referred for an ECG evaluation were: scheduled surgery, preventive examination of predisposed breeds, irregularities detected in the 
auscultatory examination, or suspicion of pathology based on a one-lead ECG performed simultaneously with the echocardiographic examination. Electrocardiographic curves were analysed with the BTL-08 Win computer system, version 6.12. Unless stated otherwise, normal values of electrocardiographic parameters were defined as proposed by Tilley and Miller (20). Numerical data were recorded in an MS Excel spreadsheet and subjected to statistical analysis.

Prevalence of arrhythmias was analysed in terms of the sex, age, body weight, and breed of the examined dogs (Figs 1 and 2). The data were organised into the following age groups: up to 1 year, 1-5 years, 6-10 years, and above 10 years of age, and into the following body weight categories: up to $1 \mathrm{~kg}, 1-10 \mathrm{~kg}, 11-25 \mathrm{~kg}$, 26-50 kg, and more than $50 \mathrm{~kg}$. Cross-breed dogs were assigned to two body weight categories: up to $25 \mathrm{~kg}$ and above $25 \mathrm{~kg}$. Based on the presence of arrhythmia, the dogs were divided into those without any abnormalities, with physiological arrhythmias (sinus pauses shorter than $3 \mathrm{~s}$, sinus arrhythmia, sinus tachycardia, and with no morphological abnormalities on echocardiographic examination) and with other (pathological) arrhythmias (2).

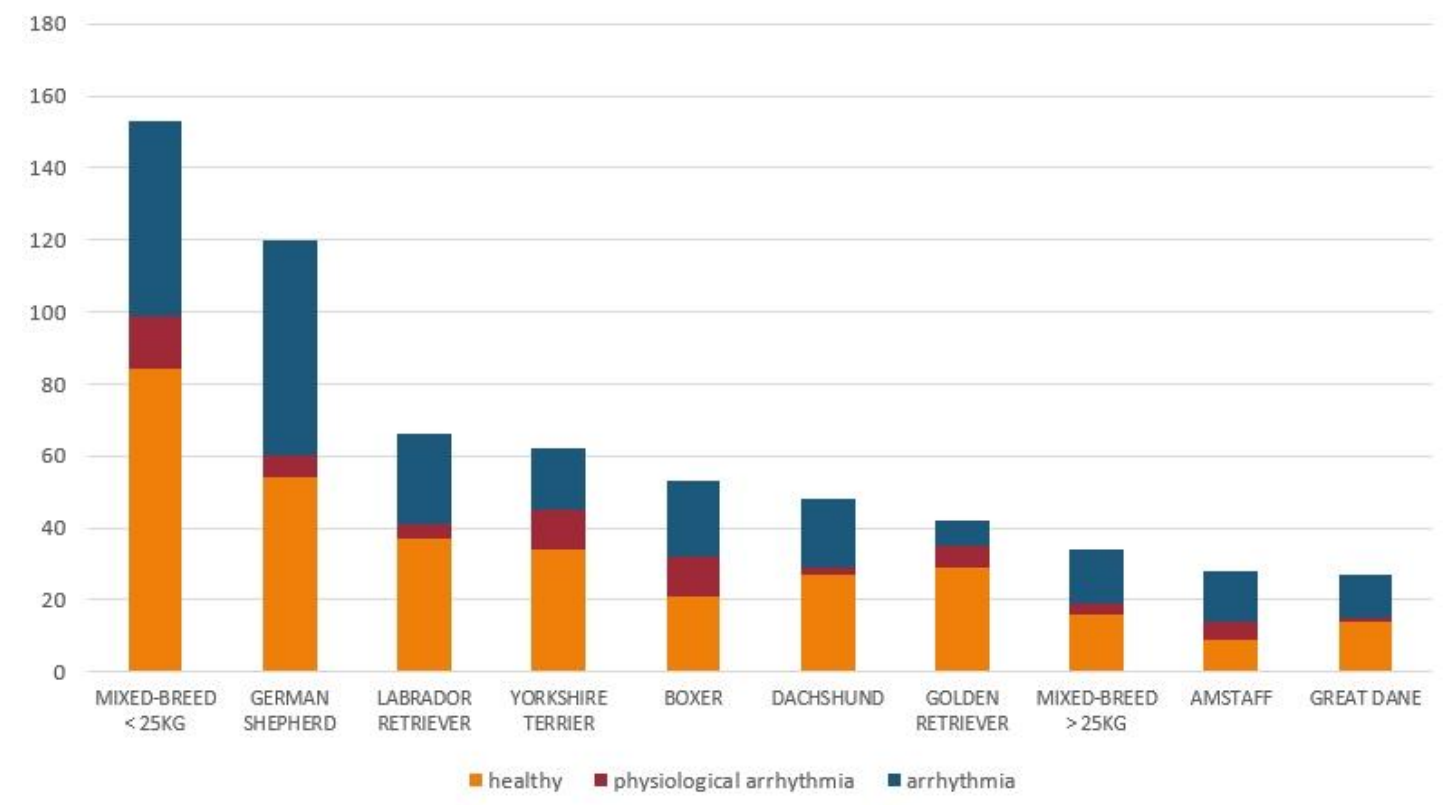

Fig. 1. Dogs of selected breeds stratified by the presence of arrhythmia

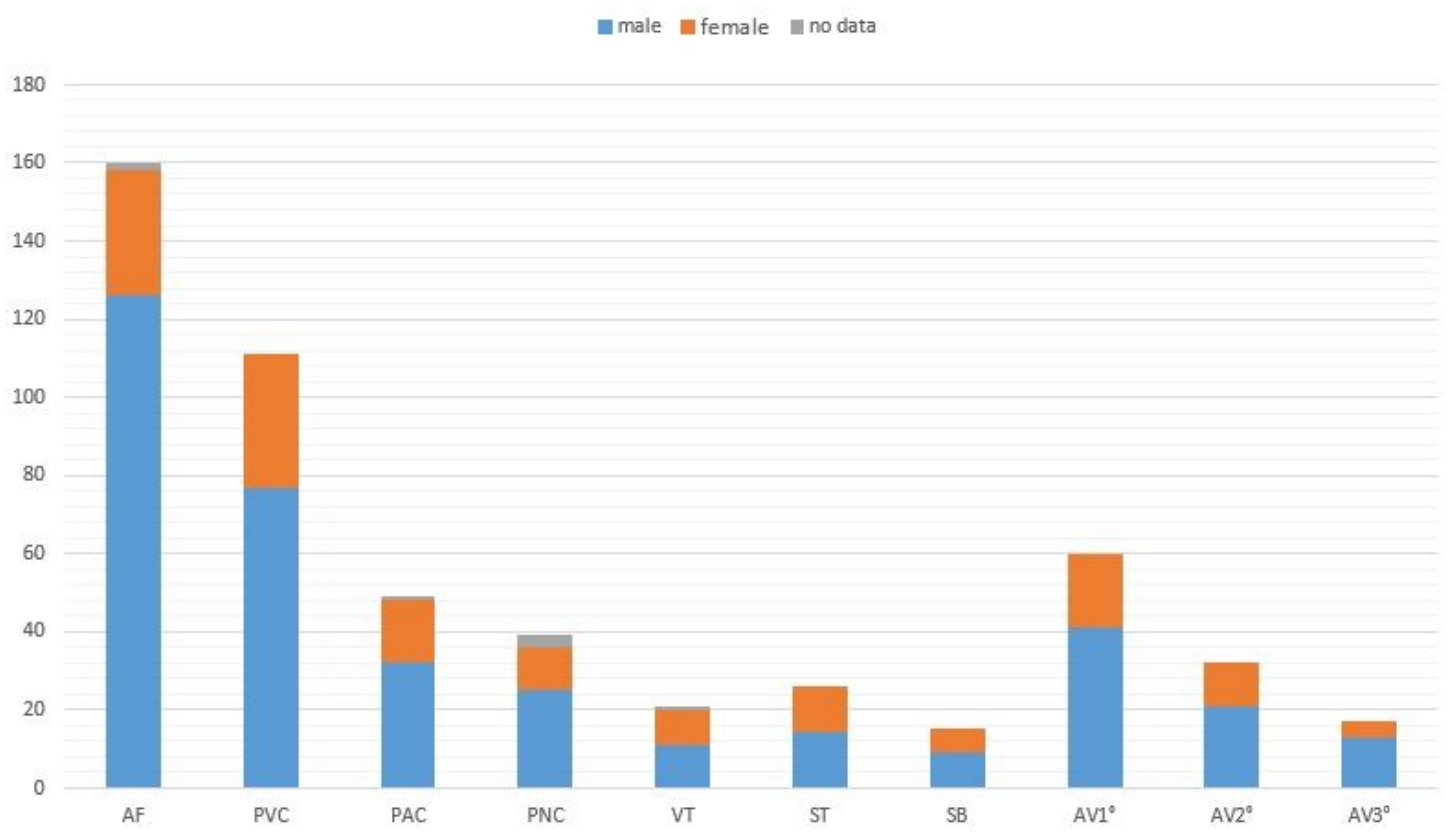

Fig. 2. Distribution of arrhythmias in correlation with sex. AF - atrial fibrillation, $\mathrm{PVC}$ - premature ventricular complex, PAC premature atrial complex, PNC - premature nodal complex, VT - ventricular tachycardia, ST - sinus tachycardia >200 bpm, SB sinus bradycardia $<70 \mathrm{bpm}, \mathrm{AV}-$ atrioventricular block 
Computer software packages, namely Statistica for Windows, version 10.0 (Statistica, USA) and PQStat ver. 1.6.2 (PQStat, Poland), were used to analyse the data. The data were compared by twoproportion $\mathrm{Z}$ test and chi-square independence test. Statistical significance was set at $5 \%$.

\section{Results}

A total of 1201 electrocardiograms from 1189 dogs were analysed. This number included 630 $(52.46 \%)$ electrocardiograms with no signs of arrhythmia, $96(7.99 \%)$ with physiological, and 475 $(39.55 \%)$ with pathological arrhythmias. The group of examined dogs included $702(59.04 \%)$ males, 466 $(39.19 \%)$ females, and $21(1.77 \%)$ animals whose sex was unspecified in medical documentation. Arrhythmias occurred significantly more often in males $(\mathrm{P}<0.001)$ taking into account their higher number in the study group. When stratified by body weight, the study group included 309 (25.99\%) dogs weighing 1-10 kg, 255 (21.45\%) dogs weighing 11$25 \mathrm{~kg}, 487$ (40.96\%) dogs weighing 26-50 kg, 89 $(7.49 \%)$ dogs weighing over $50 \mathrm{~kg}$, and $49(4.12 \%)$ dogs with unspecified body weight. The series included $46(3.87 \%)$ animals up to 1 year of age, 423 $(35.58 \%)$ aged $1-5$ years, $397(33.39 \%)$ aged 6-10 years, $306(25.74 \%)$ older than 10 years, and 17 $(1.43 \%)$ with unspecified age. The largest proportion of the examined animals were cross-breed dogs with body weight below $25 \mathrm{~kg}(\mathrm{n}=153,12.87 \%)$, followed by German Shepherds $(\mathrm{n}=122,10.26 \%)$, Labrador Retrievers $(\mathrm{n}=68,5.72 \%)$, Yorkshire Terriers $(n=63,5.3 \%)$, and Boxers $(n=60,5.05 \%)$ (Fig. 3). Other dog breeds were also represented in small numbers. The breeds of the remaining $30 \mathrm{dogs}$ $(2.52 \%)$ were unknown.

The most commonly diagnosed type of arrhythmia was atrial fibrillation (AF) $(\mathrm{P}<0.001)$ (Table 1). This arrhythmia was found in 160 out of 1189 examined dogs (13.5\% of all examined dogs and $33.7 \%$ of dogs with any pathological arrhythmia). It was significantly more often observed than all other arrhythmias $(\mathrm{P}<0.001)$. AF was found significantly more often in males $(126 / 160,78.8 \%$ of dogs with this arrhythmia and $17.9 \%$ of all male dogs in the study) $(\mathrm{P}<0001)$, in dogs aged 6-10 years $(68 / 160,42.5 \%$ of dogs with this arrhythmia and $17.1 \%$ of all dogs from this age group), in animals weighing $26-50 \mathrm{~kg}$ $(80 / 160,50 \%$ of all dogs with this arrhythmia and $16.4 \%$ of all dogs from this weight category), and in those over $50 \mathrm{~kg}$ of body weight $(29 / 160,18.1 \%$ of dogs with any arrhythmia and $32.6 \%$ of all dogs from this weight category). The most common type of arrhythmia observed concomitantly to $\mathrm{AF}$ were premature ventricular complexes (PVCs), found in 28 (17.5\%) dogs with AF. Morphology of the PVCs showed no characteristics of bundle branch block and had a marked compensatory pause. They cannot, however, be definitively differentiated from possible conduction aberrancy.

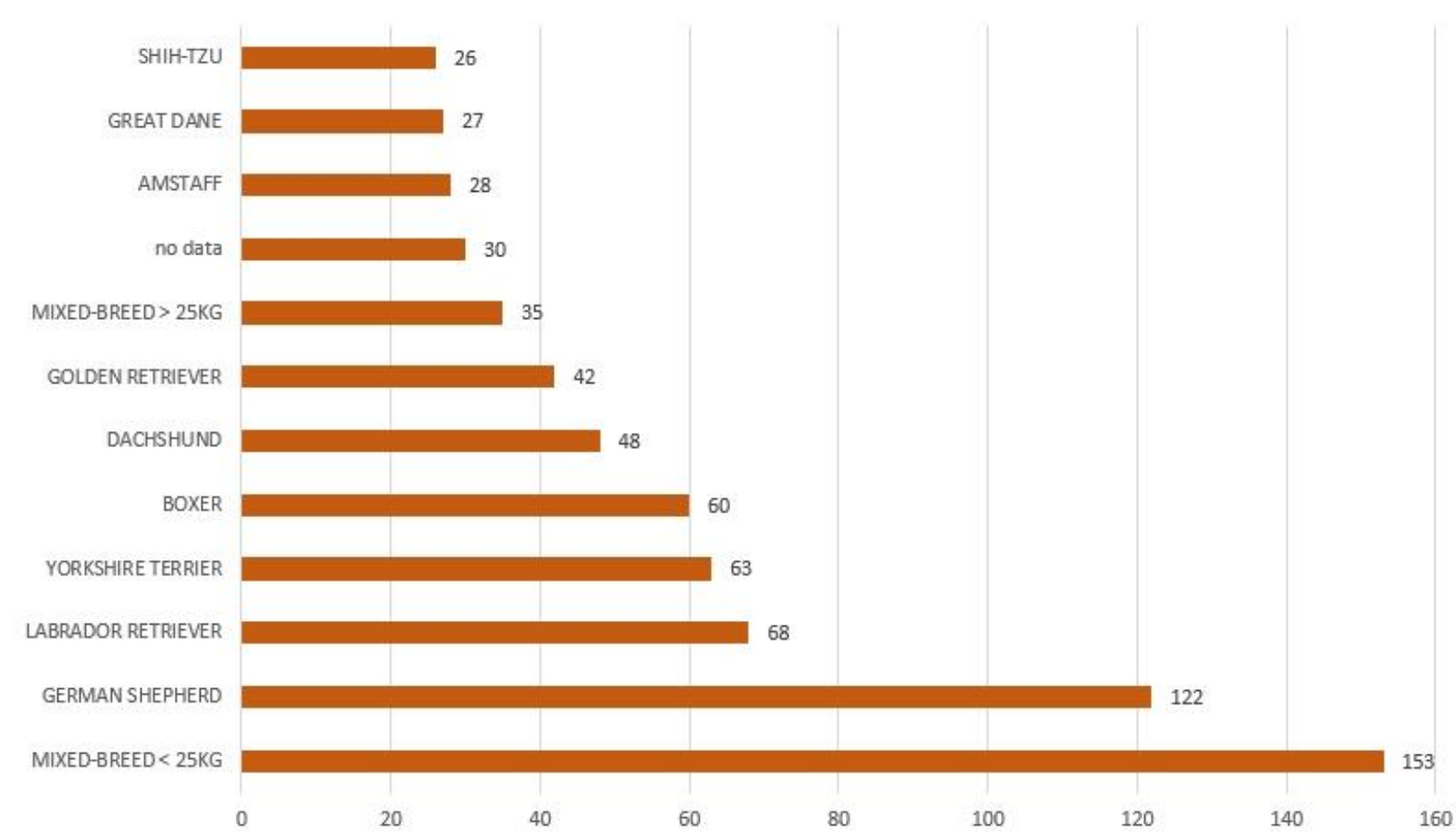

Fig. 3. Most frequent dog breeds examined in the study 
Table 1. Distribution of arrhythmia types in the examined dogs

\begin{tabular}{|c|c|c|c|c|}
\hline Type of arrhythmia & $\begin{array}{l}\text { Proportion of } \\
\text { examined dogs } \\
(\mathrm{n}=1189)\end{array}$ & $\begin{array}{l}\text { Proportion of dogs } \\
\text { with pathological } \\
\text { arrhythmia }(n=475)\end{array}$ & Males & Females \\
\hline Atrial fibrillation $(\mathrm{n}=160)$ & $13.46 \%$ & $33.68 \%$ & $78.8 \%$ & $20 \%$ \\
\hline Premature ventricular complexes $(\mathrm{n}=111)$ & $9.34 \%$ & $23.37 \%$ & $69.4 \%$ & $30.6 \%$ \\
\hline First-degree atrioventricular block $(n=60)$ & $5.05 \%$ & $12.63 \%$ & $68.3 \%$ & $31.7 \%$ \\
\hline Premature atrial complexes $(n=49)$ & $4.12 \%$ & $10.32 \%$ & $65.3 \%$ & $32.7 \%$ \\
\hline Premature nodal complexes $(n=39)$ & $3.28 \%$ & $8.21 \%$ & $64.1 \%$ & $28.2 \%$ \\
\hline Second-degree atrioventricular block $(\mathrm{n}=32)$ & $2.69 \%$ & $6.74 \%$ & $65.7 \%$ & $34.3 \%$ \\
\hline Sinus tachycardia $>200 \mathrm{bpm}(\mathrm{n}=26)$ & $2.19 \%$ & $5.47 \%$ & $53.8 \%$ & $46.2 \%$ \\
\hline Ventricular tachycardia $(\mathrm{n}=21)$ & $1.77 \%$ & $4.42 \%$ & $52.4 \%$ & $42.9 \%$ \\
\hline Supraventricular tachycardia (ex. sinus tachycardia) $(\mathrm{n}=19)$ & $1.6 \%$ & $4 \%$ & $63.2 \%$ & $36.8 \%$ \\
\hline Third-degree atrioventricular block $(\mathrm{n}=17)$ & $1.43 \%$ & $3.58 \%$ & $76.5 \%$ & $23.5 \%$ \\
\hline Sinus bradycardia $<70 \mathrm{bpm}(\mathrm{n}=15)$ & $1.26 \%$ & $3.16 \%$ & $60 \%$ & $40 \%$ \\
\hline Fusion beats $(n=7)$ & $0.59 \%$ & $1.47 \%$ & $71.4 \%$ & $28.6 \%$ \\
\hline Supraventricular escape rhythm $(n=6)$ & $0.5 \%$ & $1.26 \%$ & $50 \%$ & $50 \%$ \\
\hline Ventricular escape rhythm $(\mathrm{n}=3)$ & $0.25 \%$ & $0.63 \%$ & $100 \%$ & $0 \%$ \\
\hline Supraventricular bigeminy $(\mathrm{n}=2)$ & $0.17 \%$ & $0.42 \%$ & $100 \%$ & $0 \%$ \\
\hline Low atrial rhythm $(\mathrm{n}=2)$ & $0.17 \%$ & $0.42 \%$ & $50 \%$ & $50 \%$ \\
\hline R-on-T phenomenon $(n=2)$ & $0.17 \%$ & $0.42 \%$ & $100 \%$ & $0 \%$ \\
\hline Sinus pauses $(\mathrm{n}=131)$ & $11.02 \%$ & $27.58 \%$ & $58.9 \%$ & $41.1 \%$ \\
\hline
\end{tabular}

Supraventricular arrhythmias, namely premature atrial complexes (PACs), supraventricular tachycardia (other than sinus tachycardia), supraventricular bigeminy, and escape rhythms were found in 114 dogs (9.6\% of all examined dogs and $24 \%$ of dogs with any pathological arrhythmia). Supraventricular arrhythmias were most commonly found among males (74/114, $65 \%$ of dogs with supraventricular arrhythmias and $10.5 \%$ of all male dogs in the study), medium breeds, i.e. dogs with $11-25 \mathrm{~kg}$ body weight $(32 / 114,28 \%$ of dogs with supraventricular arrhythmias and $12.5 \%$ of all dogs from this weight category), animals at older age, i.e. in animals aged 6-10 years (41/114, 36\% of dogs with supraventricular arrhythmias and $10.3 \%$ of all dogs from this age category), and in patients older than 10 years $(38 / 114,33.3 \%$ of dogs with supraventricular arrhythmias and $12.4 \%$ of all dogs from this age category). The largest proportion of animals with supraventricular arrhythmias were Labrador Retrievers and German Shepherds (9/114, $7.9 \%$ of dogs with supraventricular arrhythmias), and the prevalence of this arrhythmia was the highest among American Staffordshire Terriers (8/28, 28.6\% of all dogs from this breed). PACs were detected in $49 / 114$ dogs $(43 \%$ of dogs with supraventricular arrhythmias, $10.3 \%$ of all dogs with any pathological arrhythmia, and $4.1 \%$ of all examined dogs). Premature nodal complexes were diagnosed in 39/114 dogs (34.2\% of dogs with supraventricular arrhythmias). A total of $19 / 114(16.7 \%)$ dogs with supraventricular arrhythmias presented with supraventricular tachycardia of nodal (9/19), atrial (2/19), or unspecified origin (8/19). Supraventricular escape rhythms were detected in $6 / 114$ patients $(5.3 \%$ of dogs with supraventricular arrhythmias). This group included 5 dogs with escape rhythms of nodal origin and 1 animal with an unspecified origin of this arrhythmia. Moreover, we found two cases of supraventricular bigeminy, and two dogs with low atrial rhythm.

Ventricular arrhythmias were found in $133 \mathrm{dogs}$ (11.2\% of all examined dogs and $28 \%$ of dogs with any pathological arrhythmia). Ventricular arrhythmias were most commonly detected in males $(90 / 133,67.7 \%$ of dogs with ventricular arrhythmias and $12.8 \%$ of all males), dogs with 26-50 kg body weight (73/133, $54.9 \%$ of dogs with ventricular arrhythmias and $15 \%$ of all dogs from this weight category), animals aged 6-10 years $(48 / 133,36.1 \%$ of dogs with ventricular arrhythmias and $12.1 \%$ of all dogs from this age group), and older animals (44/133, 33.1\% of dogs with ventricular arrhythmias and $14.4 \%$ of all dogs from this age group). German Shepherds (19/133, 14.3\% of dogs with ventricular arrhythmias) represented the largest proportion of dogs with this type of arrhythmia; however, the prevalence of ventricular arrhythmias turned out to be the highest among Irish Setters (4/10, $40 \%$ of all dogs from this breed). PVCs were the most common type of ventricular arrhythmia. It was significantly less prevalent than atrial fibrillation $(\mathrm{P}=0.008)$ but significantly more prevalent than all 
other arrhythmias $(\mathrm{P}<0.001)$. This type of arrhythmia was found in 111 dogs $(83.5 \%$ of dogs with ventricular arrhythmias and $9.3 \%$ of all examined dogs). Electrocardiograms from 45/111 (40.5\%) dogs diagnosed with this type of arrhythmia included only one PVC, whereas repeated PVCs were found in the remaining 66/111 (59.5\%) electrocardiograms. A total of $94 / 111$ dogs $(84.7 \%$ of dogs with this type of arrhythmia and $7.9 \%$ of all examined dogs) presented with single PVCs; double PVCs were found in 14/111 animals $(12.6 \%$ of dogs with PVCs and $1.2 \%$ of all examined dogs). Ventricular tachycardia was detected in 21 dogs $(15.8 \%$ of dogs with ventricular arrhythmias and $1.8 \%$ of all examined dogs), 7 of which presented sustained VT $(5.3 \%$ of dogs with ventricular arrhythmias and $0.59 \%$ of all examined dogs) and 14 non-sustained VT $(10.5 \%$ of dogs with ventricular arrhythmias and $1.2 \%$ of all examined dogs). R-on-T PVCs occurred in two dogs $(1.8 \%$ of dogs with PVCs and $0.17 \%$ of all examined dogs), and ventricular escape rhythm in three dogs $(2.7 \%$ of dogs with PVCs and $0.25 \%$ of all examined dogs). Fusion beats were found in seven dogs $(5.9 \%$ of dogs with PVCs and $0.59 \%$ of all examined dogs). No cases of ventricular fibrillation were identified.

Sinus tachycardia was defined as a rhythm of sinus origin with a heart rate $(\mathrm{HR})>200$ beats per minute (bpm), as proposed by Detweiler (4). The cutoff value for HR proposed by Tilley and Miller (16) was not considered since the information on body weight was missing in the medical documentation of some dogs. Sinus tachycardia was detected in 26 dogs (2.2\% of all examined dogs and $5.5 \%$ of dogs with any pathological arrhythmia). No association was observed between the prevalence of sinus tachycardia and the sex of the examined dogs. However, this type of arrhythmia was more frequent in dogs with $1-10 \mathrm{~kg}$ of body weight $(13 / 26,50 \%$ of dogs with sinus tachycardia and $4.2 \%$ of all dogs in this weight category) and animals older than 10 years $(11 / 26,42.3 \%$ of dogs with sinus tachycardia and $3.6 \%$ of all dogs from this age group). Cross-breed dogs with body weight $<25 \mathrm{~kg}(7 / 26$, $26.9 \%$ of dogs with sinus tachycardia and $4.6 \%$ of all dogs from this breed category) and Yorkshire Terriers $(6 / 26,23 \%$ of dogs with sinus tachycardia and $9.5 \%$ of all dogs from this breed) constituted the largest proportion of animals diagnosed with this type of arrhythmia. Prevalence of sinus tachycardia turned out to be the highest among Yorkshire Terriers $(6 / 63,9.5 \%$ of all dogs from this breed). The arithmetical mean HR in dogs with sinus tachycardia was equal to 215.4 $\pm 12.9 \mathrm{bpm}$ and the median was $215 \mathrm{bpm}$.

Sinus bradycardia was defined as a rhythm of sinus origin with a HR $<70 \mathrm{bpm}$, as proposed by Tilley and Miller (16). This type of arrhythmia was found in 15 dogs (1.3\% of all examined dogs and $3.2 \%$ of dogs with any pathological arrhythmia). No significant associations were observed between the prevalence of sinus bradycardia, age, and body weight of the animals.
No cases of sinus bradycardia were found in dogs of giant breeds, with body weights greater than $45 \mathrm{~kg}$. Sinus bradycardia was most frequent among male dogs $(9 / 15,60 \%$ of all dogs with sinus bradycardia and $1.3 \%$ of all male dogs).

Sinus pauses were detected in 131 dogs $(11 \%$ of all examined dogs and $27.6 \%$ of dogs with any pathological arrhythmia). No sex- or body weightspecific relations to sinus pauses were observed. Sinus pauses occurred most often in dogs aged 1-5 years (58/131, $44.3 \%$ of dogs with sinus pauses and $13.7 \%$ of all dogs from this age group). The largest proportion of animals with sinus pauses were dogs of brachycephalic breeds: Yorkshire Terriers (14/131, 10.7\% of dogs with this type of arrhythmia and $22.2 \%$ of all dogs from this breed), Boxers (12/131, 9.2\% of dogs with this type of arrhythmia and $20 \%$ of all dogs from this breed), American Staffordshire Terriers (8/131, 6.1\% of dogs with this type of arrhythmia and $28.6 \%$ of all dogs from this breed), French Bulldogs (5/131, 3.8\% of dogs with this type of arrhythmia and $22.7 \%$ of all dogs from this breed), and Shih-Tzus (4/131, 3.1\% of dogs with this type of arrhythmia and $15.4 \%$ of all dogs from this breed). Sinus pauses lasting less than $1 \mathrm{~s}$ were found in $29.8 \%$ of all cases of this arrhythmia, and those lasting 1-3 s and more than $3 \mathrm{~s}$ in $67.9 \%$ and $2.3 \%$ respectively.

Dogs with atrioventricular blocks constituted $9.2 \%$ of all examined animals and $22.95 \%$ of dogs with any pathological arrhythmia. First-degree atrioventricular blocks were detected in $60 \mathrm{dogs}$ (5\% of all examined dogs and $12.63 \%$ of dogs with any pathological arrhythmia). The blocks were most common among males (41/60, $68.3 \%$ of dogs with this type of arrhythmia and $5.8 \%$ of all males), dogs of large and giant breeds, i.e. with body weights of $26-50 \mathrm{~kg}$ (36/60, $60 \%$ of dogs with this type of arrhythmia and $7.4 \%$ of all dogs from this weight category) and above $50 \mathrm{~kg}(11 / 60,18.3 \%$ of dogs with this type of arrhythmia and $12.4 \%$ of all dogs from this weight category), and animals aged $1-5$ years $(20 / 60,33.3 \%$ of dogs with this type of arrhythmia and $4.7 \%$ of all dogs from this age group) and 6-10 years $(27 / 60,45 \%$ of dogs with this type of arrhythmia and $6.8 \%$ of all dogs from this age group). The largest proportion of dogs with first-degree atrioventricular blocks were German Shepherds $(9 / 60,15 \%$ of dogs with this type of arrhythmia) and the prevalence of this arrhythmia turned out to be the highest in Polish Lowland Sheepdogs $(5 / 14,35.7 \%$ of all dogs from this breed) and German Pointers (3/10, 30\% of all dogs from this breed). First-degree atrioventricular blocks represented $55 \%(60 / 109)$ of all detected atrioventricular blocks. Second-degree atrioventricular blocks were found in 32 dogs, which corresponded to $2.7 \%$ of all examined dogs and $6.74 \%$ of dogs with any pathological arrhythmia. Second-degree atrioventricular blocks were most common in males $(21 / 32,65.6 \%$ of dogs with this type of arrhythmia and $3 \%$ of all male dogs in the study), dogs of miniature breeds, i.e. with $1-10 \mathrm{~kg}$ 
body weight $(16 / 32,50 \%$ of dogs with this type of arrhythmia and $5.2 \%$ of all dogs from this weight category), and older animals, i.e. those aged 6-10 years $(14 / 32,43.8 \%$ of dogs with this type of arrhythmia and $3.5 \%$ of all dogs from this age group), and older animals $(12 / 32,37.5 \%$ of dogs with this type of arrhythmia and $3.9 \%$ of all dogs from this age group). The largest proportion of dogs with second-degree atrioventricular blocks were French Bulldogs (4/32, $12.5 \%$ of dogs with this type of arrhythmia), and the prevalence of this arrhythmia turned out to be the highest among West Highland White Terriers (3/8, $37.5 \%$ of all dogs from this breed). Second-degree atrioventricular blocks represented $29.4 \%$ of all detected atrioventricular blocks. Wenckebach periodicity was detected in 4/32 dogs with seconddegree atrioventricular block (12.5\% of dogs with this type of arrhythmia and $0.34 \%$ of all examined dogs). Third-degree atrioventricular blocks were detected in 17 dogs, which corresponded to $1.4 \%$ of all examined dogs and $3.58 \%$ of dogs with any pathological arrhythmia. Third-degree atrioventricular blocks were most common in males $(13 / 17,76.5 \%$ of dogs with this type of arrhythmia and $1.9 \%$ of all male dogs in the study). No evident age- or body weight-specific relationships to this type of arrhythmia were demonstrated. Noticeably, however, third-degree atrioventricular blocks were not detected in animals younger than one year. The largest proportion of animals with third-degree atrioventricular blocks were cross-breed dogs with body weight $<25 \mathrm{~kg}$ (4/17, $23.5 \%$ of dogs with this type of arrhythmia), and the prevalence of this arrhythmia was the highest in Dachshunds (2/48, $4.2 \%$ of all dogs from this breed). Third-degree atrioventricular blocks represented $15.6 \%$ of all detected atrioventricular blocks.

\section{Discussion}

The presented study was conducted on a large population of dogs with heart rhythm disturbances. Some arrhythmias may be physiological. Electrocardiograms from healthy individuals may provide evidence of respiratory arrhythmia resulting from fluctuations in vagal tone during the respiratory cycle. This is particularly evident in the case of brachycephalic breeds whose specific laryngeal region anatomy results in an increased vagal tone. Therefore, we considered physiological all sinus pauses that lasted no longer than $3 \mathrm{~s}$, and as such did not result in any haemodynamic disturbances (4). Another type of physiological arrhythmia is the so-called wandering pacemaker, also associated with increased parasympathetic tone (18).

We found pathological arrhythmias in $39.55 \%$ of the examined dogs, which is a higher value than reported previously. Gabriel (6) detected arrhythmias in $3 \%$ of examined dogs; similar prevalence of pathological arrhythmias has been also reported by Patterson et al. (15). In turn, Kersten et al. (10) and Tilley (19) observed arrhythmias in approximately $20 \%$ of examined animals, and Pasławska et al. (14) in $39.95 \%$. The higher prevalence of pathological arrhythmias in our series may be associated with the fact that the animals were selected for electrocardiographic examination on the basis of double clinical evaluation: by their primary veterinarians and cardiologists from our unit.

AF is characterised by the lack of P-wave, which is replaced by f-wave, as well as by an irregular and typically enhanced ventricular rhythm. AF turned out to be the most common type of arrhythmia found in our series, which is consistent with the results of previous studies, in both animals (13, 14) and humans (1). However, the prevalence of AF in our series was higher than in similarly designed previous studies conducted by Gabriel (6) (10.48\%) Tilley (19) (15.9\%), Pasławska et al. (14) (11.74\%), and Noszczyk-Nowak et al. (13) (9.7\%). In dogs, AF usually results from volume overload of the atrium and secondary atrial enlargement, or from spontaneous electrical activity in pulmonary veins (3). This may inter alia occur in dilated cardiomyopathy (DCM), a condition which typically manifests no earlier than at around eight years of age; importantly, dogs aged eight years and older showed the highest prevalence of this arrhythmia in our series. Furthermore, AF was more common in dogs of large and giant breeds, both known to be more prone to DCM. These breeds also present with the lone AF phenomenon, i.e. spontaneous $\mathrm{AF}$ non-related to any organic disease, such as atrial dilation (17). However, AF has been also found in dogs from other age groups and weight categories. In younger dogs, atrial overload may result from congenital cardiovascular diseases, whereas in older animals, it is typically associated with acquired atrioventricular valve regurgitation (11). Statistical analysis of the heart rate was not conducted in dogs with atrial fibrillation. This is partly due to lack of information regarding treatment with negative chronotropic agents.

A certain daily number of PVCs can be considered physiological. The retrospective character of this study, as well as the variable, but generally short duration of the echocardiographic examination, did not allow us to distinguish between animals with physiological and pathological PVCs; therefore, all PVCs identified in this study were by default classified as pathological. Published data on the prevalence of PVCs vary: according to Pasławska et al. (14) they occur in $8.9 \%$ of dogs with arrhythmias, whereas Gabriel (6) and Tilley (19) found them in $34.67 \%$ and $25.5 \%$ of their patients, respectively. The study conducted by the latter author included the largest number of patients of all the mentioned authors, and its results seem to be most consistent with our findings. Detweiler (5) observed PVCs in $0.6-1 \%$ of clinically normal Beagle dogs. However, none of the papers mentioned above 
presented the exact character of this arrhythmia. In our study, PVCs turned out to be the most frequent in breeds predisposed to DCM. The latter is more prevalent in male dogs and animals older than five years, which is also consistent with the results of our study. In turn, Boxers are predisposed to arrhythmogenic right ventricular cardiomyopathy which is characterised by the occurrence of ventricular arrhythmias resulting from fatty infiltration of the right ventricular myocardium. The high prevalence of PVCs in Irish Setters should probably be considered a statistical artefact, owing generally to the low representation of this breed in our series.

To our knowledge, none of the previous studies dealt with the prevalence of the other ventricular arrhythmias adopted as a subject of our analysis. Due to the low number of these arrhythmias, we did not stratify their prevalence in terms of breed, sex, and age.

Physiological sinus tachycardia can be interpreted as a consequence of short-term catecholamine release, representing a stress response to electrocardiographic examination. However, HR >200 bpm, despite the sinus origin, should be always classified as pathological owing to its haemodynamic consequences: a decrease in cardiac output and fall in myocardial oxygen supply with a simultaneous increase in oxygen demand. If observed at rest, such accelerated heart rate may reflect sympathetic activation during the course of end-stage heart failure.

Sinus bradycardia is rarely observed in a routine clinical practice. Although it may occur physiologically in athletic dogs of giant breeds, we did not document this phenomenon in our series. In smaller dogs, sinus bradycardia may result from an increased vagal tone or toxaemia, or develop secondarily to sick sinus syndrome (SSS).

Sinus pauses are typically observed in brachycephalic breeds. They may occur secondary to fluctuations in vagal tone associated with the respiratory cycle. Such fluctuations are particularly evident in dogs with brachycephalic syndrome due to their increased respiratory effort, irritation of laryngeal region, and parasympathetic stimulation. In our study, all pauses lasting up to $3 \mathrm{~s}$ were considered a physiological manifestation of the phenomena mentioned above, and only longer pauses were qualified as pathological. Such long pauses may occur secondarily to SSS or digitalis toxicity. Most animals with bradycardia were referred for $24 \mathrm{~h}$ electrocardiographic Holter monitoring to determine circadian fluctuations in HR and identify potential signs of SSS; the latter was eventually confirmed in eight patients.

Gabriel (6) was the only author who examined the prevalence of atrioventricular blocks; she found them in $19.35 \%$ of dogs with arrhythmia, which is consistent with our findings. To the best of our knowledge, none of the previous studies examined the prevalence of other blocks nor determined detailed characteristics thereof. In our study, first-degree atrioventricular blocks were most often detected in dogs whose age was consistent with that at which hypothyroidism is typically diagnosed, and it was the latter condition which was probably the most common cause of this arrhythmia in our series. However, due to the retrospective character of this study, we were unable to verify if the atrioventricular blocks found in some dogs were associated with previous pharmacotherapy. Based on their medical histories, drug-induced ventricular blocks might have occurred in four dogs; the remaining nine dogs were eventually diagnosed with hypothyroidism and one of them with congenital atrioventricular block. High-grade atrioventricular blocks are usually claimed to be idiopathic and associated with fibrosis of the conduction system or myocarditis (8).

To the best of our knowledge, PACs were the only type of supraventricular arrhythmia the prevalence of which had been a subject of previous studies. However, available data on the prevalence of PACs are inconclusive. Pasławska et al. (14) found them in $5.45 \%$ of dogs with arrhythmia and Tilley (19) in $28.53 \%$; in turn, the prevalence of PACs in our study was $10.32 \%$.

Due to the retrospective character and long period covered by the analysis, we were unable to retrieve complete medical histories of all the examined dogs, specifically the data on previous treatments, comorbidities, additional tests performed at other centres, and whether a patient was a primary one or sent for evaluation by a third-party. We are aware that because the data were obtained only in one cardiology unit, they may not adequately illustrate the real population. We also cannot provide exact information on how many patients overall were evaluated in the Cardiology Unit at the same period due to lacking medical documentation. There is no available information on statistical analysis of breeds in Poland, so this kind of data could not be compared to the results of this study.

In conclusion, supraventricular arrhythmias were dominant, with atrial fibrillation being the most common problem. Male dogs were more prone to heart rhythm disturbances. A significant number of healthy dogs presented physiological arrhythmias.

Conflict of Interests Statement: The authors declare that there is no conflict of interests regarding the publication of this article.

Financial Disclosure Statement: This research was supported by statutory funding for research and development activity by the Polish Minister of Science and Higher Education assigned to the Faculty of Veterinary Medicine, Wroclaw University of Environmental and Life Sciences.

Animal Rights Statement: None required. 


\section{References}

1. Allessie M.A., Boyden P.A., Camm A.J., Kleber A.G., Lab M.J., Legato M.J., Rosen M.R., Schwartz P.J., Spooner P.M., Van Wagoner D.R., Waldo A.L.: Pathophysiology and prevention of atrial fibrillation. Circulation 2001, 103, 769-777.

2. Bliss S.P., Atkins C.E.: ECG of the month. Sinus arrhythmia in a dog. J Am Vet Med Assoc 1997, 211, 1126-1127.

3. Chen Y.J., Chen S.A., Chang M.S., Lin C.I.: Arrhythmogenic activity of cardiac muscle in pulmonary veins of the dog: implication for the genesis of atrial fibrillation. Cardiovasc Res 2000, 48, 265-273.

4. Detweiler D.: The dog electrocardiogram: A critical review. In: Comprehensive Electrocardiology: Theory and Practice in Health and Disease, edited by Macfarlane P.W., Lawrie T.D.V., Pergamon Press, New York, 1993, p. 1883.

5. Detweiler D.: The use of electrocardiography in toxicological studies with Beagle dogs. In: Cardiac Toxicology, edited by Balazs T., CRC Press, Boca Raton, 1981, pp. 33-82.

6. Gabriel A.: Zuroralen und parenteralen Therapie von Herzrhythmusstörungen des Hundesmit Propafenon. Doctoral Thesis. Hannover: Tierärztliche Hochschule; 1992.

7. Janus I., Noszczyk-Nowak A., Nowak M., Cepiel A., Ciaputa R., Pasławska U., Dzięgiel P., Jabłońska K.: Myocarditis in dogs: etiology, clinical and histopathological features (11 cases: $2007-$ 2013). Ir Vet J 2014, 67, 28.

8. Kersten U., von Winterfeldt K., Brass W.: Statistische Erhebungen uber Herzkrankheiten beim Hund. Kleintierpraxis $1969,14,45-47$
9. Ljungvall I., Rishniw M., Porciello F., Ferasin L., Ohad D.G.: Murmur intensity in small-breed dogs with myxomatous mitral valve disease reflects disease severity. J Small Anim Pract 2014, $55,545-550$

10. Noszczyk-Nowak A., Pasławska U., Zyśko D., Gajek J., Nicpoń J., Hebel M.: Atrial fibrillation in dogs. Med Weter 2008, 64, 686-690.

11. Pasławska U., Noszczyk-Nowak A., Okrąglicka E., Nicpoń J.: Analysis of heart arrhythmia in dogs examined between 1996 and 2001 in the Clinic of Horse, Dog and Cat Diseases at the Faculty of Veterinary Medicine of Wrocław Agricultural University. Med Weter 2004, 60, 1191-1195.

12. Patterson D.F., Detweiler D.K., Hubben K., Botts R.P.: Spontaneous abnormal cardiac arrhythmias and conduction disturbances in the dog. A clinical and pathologic study of 3000 dogs. Am J Vet Res 1961, 22, 355-369.

13. Saponaro V., Tursi M., Migliorini F., Crovace A.: ECG of the month. Cardiac arrhythmia associated with atrial fibrosis and a heart tumor. J Am Vet Med Assoc 2013, 243, 489-491.

14. Tanner J.C., Lake-Bakaar G.A., Kittleson M.D.: ECG of the month. Arrhythmia. J Am Vet Med Assoc 2013, 243, 637-639.

15. Tilley L.: EKG bei Hund und Katze: Grundlagen. Auswertung und Therapie. Hannover, Schlütersche Verlagsanstalt, 1989, p. 60.

16. Tilley L., Miller S.: Manual of Canine and Feline Cardiology. Philadelphia, W. B. Saunders, 1995, p. 54.

17. Ulloa H.M., Houstin B.J., Altrogge D.M.: Arrhythmia prevalence during ambulatory electrocardiographic monitoring of beagles. Am J Vet Res 1995, 56, 275-281. 